\title{
COVID-19 and Elective Surgery
}

\section{Practical Tips for a Safe, Successful, and Sustainable Reboot}

\author{
Zhen Chang Liang, MBBS, MRCS, DipSpMed, PhD, MBA, * Mark Seng Ye Chong, BMBS, BMedSc, FRCS, \\ Gabriel Ka Po Liu, MBBS, MSc, FRCS, * Alejandro Gonzalez Della Valle, MD, $\dagger$ Dahui Wang, MD, PhD, \\ Xuemin Lyu, MD, PhD, § Chih-Hung Chang, MD, PhD, FICS, $\uparrow||$ Tae-Joon Cho, MD, ** \\ Steven B. Haas, MD, MPH, $\dagger$ Dale Fisher, MBBS, FRACP, ${ }^{* *} \|$ Diarmuid Murphy, MBBS, FRCS, ${ }^{*}$ \\ and James Hoi Po Hui, MBBS, MD, FRCS*
}

Keywords: COVID-19, elective surgery, reboot, safe, successful, sustainable

(Ann Surg 2021;273:e39-e40)

$T^{\prime \prime}$ he COVID-19 pandemic has thrown surgical systems worldwide into unprecedented disarray. Elective surgery has stopped to preserve scarce healthcare resources and aid in redeployment of staff to areas where they are most needed. Some surgical disciplines, for example, Orthopaedics have been affected more than others. The COVID-19 crisis is anticipated to remain with us for an extended period of time. An indefinite suspension of elective surgery is not practical as this would have far-reaching and deleterious implications on patient care. Equally important, the financial sustainability of healthcare systems would be severely stressed. In this perspective, we provide 7 practical tips, to be considered in stepwise progression, for healthcare systems looking to reboot their elective surgeries in a safe, successful, and sustainable manner.

From the *Department of Orthopaedic Surgery, National University of Singapore, National University Health System, Singapore; $†$ Hospital for Special Surgery, New York, New York; †National Children's Medical Center \& Children’s Hospital of Fudan University, Shanghai, People's Republic of China; $\S$ Department of Orthopaedic Surgery, Beijing Jishuitan Hospital, Xicheng, People's Republic of China; † Department of Orthopaedic Surgery, Far Eastern Memorial Hospital, New Taipei City, Taiwan; Graduate School of Biotechnology and Bioengineering, Yuan Ze University, Taoyuan, Taiwan; ||Division of Infectious Disease, National University Hospital, Singapore; and ${ }^{* *}$ Division of Paediatric Orthopaedics, Seoul National University College of Medicine, Seoul National University Children's Hospital, Seoul, Korea.

凶zhen_chang_liang@nuhs.edu.sg.

The decision to restart elective surgery amid this COVID-19 pandemic is a challenging, tricky but ultimately necessary one. The key to any successful reboot plan lies in its safety and sustainability. To best address and answer this question, we have sought to obtain diverse views from healthcare leaders and understand varying practices within healthcare institutions in different parts of the world. This accounts for the number of authors included in this manuscript. It is hoped that this diversity of views will allow readers to have a more wholesome understanding/grasp of the issue at hand.

The specific contributions of the authors are as follows: Zhen Chang Liang: idea conception, drafting, and approval of final manuscript; Mark Seng Ye Chong: idea conception, drafting, and approval of final manuscript; Gabriel Ka Po Liu: idea conception, drafting, and approval of final manuscript; Alejandro Gonzalez Della Valle: drafting and approval of final manuscript; Dahui Wang: drafting and approval of final manuscript; Xuemin Lyu: drafting and approval of final manuscript; Chih-Hung Chang: drafting and approval of final manuscript; Tae-Joon Cho: drafting and approval of final manuscript; Steven B. Haas: drafting and approval of final manuscript; Dale Fisher: drafting and approval of final manuscript; Diarmuid Murphy: drafting and approval of final manuscript; James Hoi Po Hui: supervision, drafting, and approval of final manuscript.

No sources of support or funding are reported for this work.

The authors declare no conflict of interest.

Copyright (C) 2020 Wolters Kluwer Health, Inc. All rights reserved.

ISSN: 0003-4932/20/27302-0e39

DOI: $10.1097 /$ SLA.0000000000004091

\section{KNOW WHERE YOU ARE ON THE EPIDEMIC CURVE}

Work with your local health authorities, hospitals, and epidemiologists. Understand where your county/state/country is on the epidemic curve. ${ }^{1}$ Electives should preferably only start when the epidemic curve is flat, with no upsurge/spikes in new infections and when community spread is minimal or controlled. This is to avoid inadvertent introduction of COVID 19 into an over-burdened hospital system. Take time to monitor the local situation before committing to restarting elective work. This will allow for any reporting delays which can mislead us into believing an outbreak is over before it truly is. History is a great teacher. The 1918 flu pandemic comprised of 3 waves; the second wave being more deadly than the first. ${ }^{2}$ We would do well to learn from the lessons of our past.

\section{ENSURE ACCESS TO RELIABLE AND ACCESSIBLE COVID-19 SCREENING}

One of the major difficulties in controlling COVID-19 is of spread through asymptomatic patients. No amount of screening for temperature, upper respiratory tract symptoms, or recent travel will reduce this risk. A prerequisite for return to elective surgery is the availability of a reliable screening test(s) for COVID -19. This should form the cornerstone of a safe and successful reboot. Screening should not overtly burden local laboratories. The gold standard in screening is the Reverse transcription polymerase chain reaction (RTPCR) based naso/oro-pharyngeal swab. This evaluates for the presence of viral RNA, has a sensitivity and specificity of $>90 \%$ and is quintessential for the detection of acute, even asymptomatic COVID19 infection. ${ }^{3}$ These tests may be harder to come by given acute shortages of key reagents as the pandemic wages on.

Rapid serology tests that detect the presence of COVID-19 antibodies may be less reliable given that results depend on the individual's immune response. Acute, asymptomatic, and milder infections may potentially be missed, putting the safety of surgical teams and healthcare personnel at risk. ${ }^{3}$ It is also unknown if the presence of antibodies confer immunity and protect against re-infection. Some hospitals in Beijing have adopted a combination of serology tests and computed tomography chest to screen elective patients. In South Korea, patients are screened using only RT-PCR swabs within 1-2 days of their elective procedures. It is imperative to note that the longer the lag time between screening and surgery, the higher the risk for error.

Healthcare workers must have ready access to COVID-19 testing. Defined protocols must be established, before elective surgery "reboot," which enables ease of contact tracing and quarantine measures for any symptomatic/infected staff and their close contacts. Contingency plans should also be developed for "worst case scenarios," involving outbreaks affecting staff and patients in elective wards. 


\section{UNDERSTAND HOSPITAL RESOURCES}

Screening is of little value without adequate hospital infrastructure and manpower support. Surgery requires a team effort, and a successful reboot plan requires a coordinated effort amongst all stakeholders, from hospital leadership right down to logistical staff. Hospitals should plan for pandemic-ready Operating Rooms (ORs), an adequate number of isolation beds, retrofitted elective wards to allow for safe distancing, a steady Personal Protective Equipment (PPE) supply, and rejuvenated trained staff. Importantly, a ready supply of anaesthetic drugs needs to be secured before electives can reasonably resume. This may be challenging given anticipated shortages with the increasing number of ventilated COVID patients. ${ }^{4}$ Elective work should not, under any circumstances, compromise care for patients with emergent/urgent conditions. Hospitals must not be left surviving on crisis mode. The resources must be available for preoperative, intraoperative, and postoperative surgical precautions to be undertaken as strictly as possible to prevent cross-contamination and nosocomial infection. ${ }^{5}$

\section{ENSURE APPROPRIATE CASE SELECTION AND PRIORITIZATION}

Elective cases must be prioritized and this should preferably be coordinated by a centralized committee. Principles of prioritization $^{6}$ include clinical urgency, extent of utility of hospital resources, for example, intensive care unit beds, severity of disabling symptoms, history of previous cancellations, and patient demographics. Objective scoring systems such as the Medically Necessary TimeSensitive (MeNTS) instrument can be useful for this purpose. ${ }^{7}$ Ideal priority cases to kickstart the rebooting process are patients who are young (few comorbidities) with disabling symptoms in whom an uncomplicated and early discharge is anticipated.

\section{OPTIMIZE THE PATIENT'S POST-DISCHARGE PLANNING}

Agree on a rehabilitation plan with the patient before surgery. Patients should ideally require minimal rehabilitation, or undergo rehabilitation protocols that can be easily supervised at home via online platforms. Technology should be leveraged for this purpose. Avoid discharging patients to rehabilitation facilities or nursing homes. They can be potential hotbeds for COVID spread and transmission. ${ }^{8}$

\section{START SMALL, STAY CURRENT, AND BE ADAPTABLE}

Start small and aim for a gradual ramp-up in caseload and services. A 10\%-20\% capacity goal is reasonable in the initial reboot phase. Begin with ambulatory cases before considering inpatient admissions. Avoid overlapping surgery given its potential risk of cross-contamination. Scale up or down accordingly depending on the local COVID situation. Be prepared to tap on and off the brakes, until an effective vaccine is developed or herd immunity has been achieved. ${ }^{9}$ Stay current with the latest COVID-19 literature, monitor your outcomes, for example, complication rates and adapt your practices accordingly.

\section{BE PREPARED FOR ROADBLOCKS AHEAD}

Medical hurdles aside, financial and legal roadblocks can easily derail any plans for a successful and sustainable reboot. Be mindful of increased costs against a reduced bottom line. With the surge in demand, costs can quickly escalate from PPE requirements, COVID screening, tests and the need for repeated facility sanitization and disinfection. ${ }^{10}$ These additional costs may not necessarily be covered by existing insurance or even by governmental aid. Furthermore, departments may come under pressure from hospital administrators to ramp up their elective work as this is the main source of revenue for the hospital. Rebooting should follow a logical stepwise progression. It must be acknowledged that recommencing elective work is not without risks and potential legal ramifications. What if a patient or staff contracts a nosocomial infection and decides to seek legal redress? Pre-planning should help minimize these risks. Again, the challenge is not with re-initiating the reboot. The key lies in its safe sustainability.

Restarting elective surgery is an eventuality which we will all have to embrace. Rather than sweep it under the carpet, we need to begin facilitating the tough conversations to ensure its success. The key challenges lie in deciding on the optimal timing, working within limitations, at the same time ensuring the safety of healthcare workers and patients involved. Start small and stay nimble. Be prepared for potential financial and legal setbacks. Reboot safely, and see you on the other side.

\section{REFERENCES}

1. Prevention CfDCa. Interpretation of Epidemic Curves During Ongoing Outbreak Investigations. 2020. Available at: https://www.cdc.gov/foodsafety/outbreaks/investigating-outbreaks/epi-curves.html. Accessed May 19, 2020.

2. Prevention CfDCa. 1918 Pandemic Influenza: Three Waves. 2020. Available at: https://www.cdc.gov/flu/pandemic-resources/1918-commemoration/threewaves.htm. Accessed May 19, 2020.

3. Patel R, Babady E, Theel ES, et al. Report from the American Society for Microbiology COVID-19 international summit, 23 march 2020: value of diagnostic testing for SARS-CoV-2/COVID-19. mBio. 2020;11:e00722-e00820.

4. Brennan Z. FDA Reports Shortage of Sedation Drug Used for Putting COVID19 Patients on Ventilators. 2020. Available at: https://www.raps.org/news-andarticles/news-articles/2020/4/fda-reports-shortage-of-sedation-drug-used-forput. Accessed May 19, 2020.

5. Liang ZC, Chong MSY, Sim MA JLL, et al. Surgical considerations in COVID-19 patients: what orthopaedic surgeons should know. J Bone Joint Surg. 2020. 10.2106/JBJS.20.00513. doi:10.2106/JBJS.20.00513.

6. Surgeons ACo. Joint Statement: Roadmap for Resuming Elective Surgery After COVID-19 Pandemic. 2020. https://www.asahq.org/about-asa/newsroom/newsreleases/2020/04/joint-statement-on-elective-surgery-aftercovid-19-pandemic

7. Prachand VN, Milner R, Angelos P, et al. Medically-necessary, time-sensitive procedures: a scoring system to ethically and efficiently manage resource scarcity and provider risk during the COVID-19 pandemic. J Am Coll Surg. 2020. S1072-7515(20)30317-3. doi:10.1016/j.jamcollsurg.2020.04.011.

8. Dosa D, Jump RLP, LaPlante K, et al. Long-term care facilities and the coronavirus epidemic: practical guidelines for a population at highest risk. $J$ Am Med Dir Assoc. 2020;21:569-571.

9. Cyranoski D. 'We Need to be Alert': Scientists Fear Second Coronavirus Wave as China's Lockdowns Ease. 2020. Available at: https://www.nature.com/ articles/d41586-020-00938-0. Accessed May 19, 2020.

10. Daniella Diaz GSaCA. Protective Equipment Costs Increase Over $1000 \%$ Amid Competition and Surge in Demand. 2020. Available at: https://edition.cnn.com/2020/04/16/politics/ppe-price-costs-rising-economy-personalprotective-equipment/index.html. Accessed May 19, 2020. 